\title{
HEINE O LA DUCTILIDAD DEL ROMANTICISMO
}

\author{
HEINE OR THE DUCTILITY OF ROMANTICISM
}

\section{Daniel Moreno Moreno}

Heinrich Heine, Sobre bistoria de la religión y la filosofía en Alemania. La escuela romántica. Traducción de Manuel Sacristán. Introducción de Manuel Garrido. Estudio crítico a cargo de Manuel Sacristán. Tecnos, Madrid, 2015, 390 pp.

Resulta difícil que un libro de aspecto tan modesto como el arriba reseñado concentre tanta intensidad; y, si se me apura, tanto simbolismo. Téngase en cuenta que los dos textos recogidos están firmados por H. Heine y que los dos espadas que lo flanquean son nada menos que Manuel Garrido y Manuel Sacristán, máximos representantes de dos de los movimientos filosóficos más relevantes de renovación surgidos en los años sesenta del pasado siglo: el que miraba a lo anglosajón y norteamericano, liberal en lo político - la filosofía analítica, por simplificar - y el que miraba a lo alemán, incluso a lo soviético, afín a cualquier revolución - la filosofía marxista, en breve-. Compartían ambos, curiosamente, además del nombre de pila y el año de nacimiento, su interés por la lógica matemática y, lo que contribuye a desdibujar fáciles maniqueísmos, una curiosidad intelectual literalmente sin límites, nada dogmática y con múltiples puntos de contacto. Por debajo de sus diferencias intelectuales y de su famoso enfrentamiento por una cátedra de lógica, les unía el común interés por abrir el panorama filosófico español-y seguramente el político- a lo que ocurría fuera de la España autárquica, no sólo en lo económico, sino en lo religioso y en lo cultural. El esfuerzo era de tal calibre, proporcional al hueco que había que llenar, que acaso eso explique que, en ambos pensadores, una parte sustancial de su obra se encuentre dispersa en prólogos y presentaciones a traducciones de los autores más diversos. Un buen ejemplo de estas características —inesperados puntos en común e intensidad filosófica destilada en pequeños textos- es el libro Heinrich Heine, Sobre historia de la religión y la filosofía en Alemania. La escuela romántica, en una edición que conjunta la traducción y el estudio crítico de Manuel Sacristán —publicados originalmente en 1964-, y la decisión de Manuel Garrido de 
incluirlo en su prestigiosa colección "Los esenciales de la filosofía", junto a una breve introducción a su cargo ${ }^{1}$.

Esta edición constituye una excelente ocasión para volver a leer la mejor prosa de Heine - aguda, irónica, iluminada - y abordar un periodo histórico donde se sembraron las semillas de las que brotó la modernidad, lo contemporáneo. Su autor, nacido en la confluencia del pequeño río Düssel con el gran Rin, llamado la primera parte de su vida Harry - a la inglesa, puesto que sus padres, judíos ilustrados, admiraban Inglaterra - y la segunda, al bautizarse como cristiano protestante, Heinrich, su nombre germanizado, fue un estupendo intérprete entre dos naciones llamadas a mantener una relación difícil. Francia, tras su revolución y las luchas napoleónicas, ya estaba en construcción, alternando entre república y monarquía, y con París siempre a la cabeza; mientras tanto, Alemania sólo nombraba en ese momento una comunidad idiomática que flotaba por encima de múltiples unidades políticas, diversas tradiciones culturales y dos religiones dominantes, la protestante y la católica. La patria de Heine, Renania, cumplió el papel de vía de comunicación, de los ejércitos tanto como de las diversas influencias culturales, y la misma vida y obra de Heine urdieron tal urdimbre entre las dos orillas del Rin que el famoso poeta romántico bien merece el título de Hermes reencarnado. Él fue muy consciente de las dos Francias que luchaban entre sí, así como de las dos Alemanias también en guerra civil; de modo que da que pensar el hecho de que, si hubiera sido tan longevo como su odiado-admirado Goethe, habría asistido a la guerra franco-prusiana de 1870.

Dado que Manuel Garrido falleció el 8 de enero de 2015, esta publicación ha resultado ser la última en la que trabajó. Esa circunstancia explica que el libro esté dedicado "A la memoria de los filósofos Manuel Sacristán (1925-1985), Manuel Garrido (1925-2015)". Loable detalle por parte de los editores que, no obstante, queda algo deslucido por el evidente descuido a la hora de revisar las pruebas; testimonio de lo cual son las tantas pequeñas erratas, y no tan pequeñas ${ }^{2}$, como

\footnotetext{
${ }^{1}$ Por mor de la exactitud, hay que recordar que los dos textos de Heine, en cuidada edición de José Carlos Velasco sobre la traducción de Manuel Sacristán, han visto recientemente la luz en Alianza Editorial, en 2008 Sobre la bistoria de la filosofía y la religión en Alemania y en 2010 La escuela romántica.

${ }^{2}$ Es realmente grave que en la página 116 primera línea se salten dos páginas de la edición de Sacristán. Así la leyenda que Heine recoge al comienzo del libro tercero sobre el mecánico inglés que construyó un hombre sin alma queda sin sentido.
} 
presenta el texto final, algo que al profesor Garrido, siempre tan meticuloso, le habría seguramente molestado.

Mi modo de abordar un libro tan intenso dibujará una espiral en sentido inverso al temporal. Así, empezaré comentando el último texto escrito, la introducción de Manuel Garrido, titulada "La otra cara de Heine" y fechada en "Otoño de 2014"; de ahí pasaré al estudio crítico de Manuel Sacristán, titulado "Heine, la consciencia vencida" y fechado en "Enero de 1963". Los dos textos de Heine fueron escritos en los primeros años treinta del siglo XIX y publicados alternativamente en francés y en alemán, en varias versiones; la versión que se ofrece da para los dos textos la fecha de 1835, aunque las distintas versiones abarcan el periodo de 1833 a 1836.

Lo que acaso llame en primer lugar la atención es que un autor conocido como poeta encuentre su sitio en una colección llamada "Los esenciales de la filosofía" y ocupe un lugar junto a pensadores clásicos como Aristóteles, Hume o Santayana. De ahí que Manuel Garrido titule su introducción "La otra cara de Heine", dado que el libro recoge dos textos en prosa de la faceta seguramente menos conocida de Heine: el Heine que ejerce de cronista en francés de las novedades editoriales publicadas en alemán, lengua que en Francia pocos conocen; aunque se da la paradoja de que las crónicas se publican también en alemán, circunstancia que le permite a Heine dirigirse alternativamente a los franceses y a los alemanes; es más, le permite lucir su habilidad para una escritura doble, escrita, como si dijéramos, simultáneamente con la mano derecha y con la mano izquierda, y con un ojo mirando en cada dirección. Contribuyó así decisivamente al proceso de decantamiento de "lo francés" frente a "lo alemán", al elegir, entre los múltiples y enfrentados elementos presentes en cada bloque cultural, los aspectos que él consideraba más característicos de cada uno. De ahí que los juicios heineanos hayan adquirido el carácter de clásicos —en el sentido de que cada vez que se visitan aportan nueva luz al lector- y Heine ocupe dignamente su lugar al lado de otros pensadores esenciales.

Para el profesor Garrido, a Heine le cuadran bien los epítetos "superdotado para el debate filosófico, político y periodístico" (p. 11), "precoz, agudo y descarado" (p. 12) y habla de "su genial y escandalosa idiosincrasia" (id.); a su reconstrucción de la filosofía alemana, no obstante, la califica de "original y revolucionaria, aunque en parte desviada" (p. 12), y a su figura en general le encaja el siguiente juicio; "la genialidad de Heine tuvo siempre por compañeras una cierta incoherencia de pensamiento y una dosis nada homeopática de venalidad" (p. 18). Como intérpretes de la dúctil posición heineana, Garrido recuerda la de Marcuse y la de Adorno, 
publicadas al cumplirse en 1956 el primer centenario de su nacimiento ${ }^{3}$, aunque prefiere la de Manuel Sacristán, "Heine, la consciencia vencida", ensayo al que llama "un excelente análisis crítico del pensamiento filosófico-literario y de la praxis política de Heine, que no tiene rival en la bibliografía filosófica y literaria española de los últimos cincuenta años" (p. 18). Garrido pasa a primer plano, además, la paradoja siguiente: cómo es que el poeta romántico casi por antonomasia se presenta a sí mismo como antirromántico, como el enterrador del romanticismo.

El estudio del profesor Sacristán, con todo, intenta responder a otra cuestión: cómo es que el poeta demócrata y revolucionario, también casi por antonomasia, se siente finalmente asustado por la revolución de 1848 y se hace conservador. Su enfoque, además, es de gran angular, dado que originalmente el ensayo era la introducción al voluminoso libro Obras de Heine publicado en 1964, por lo que no se centra específicamente en los dos ensayos de Heine ahora rescatados ${ }^{4}$. De ahí el pormenorizado e informado repaso a la vida del poeta renano, en base fundamentalmente a sus cartas, que ocupa la primera mitad de su escrito, casi con ánimo de censor.

Con gran sentido de la plasticidad, el profesor Sacristán parte del momento en que Heine, a los 34 años de edad — casi la misma que debía de tener él mismo cuando comenzó la magna tarea de traducirlo— cruza el Rin camino de París. Atrás quedaban sus ya famosos poemas - el internacionalmente conocido Buch der Lieder [Libro de canciones] (1827), en realidad una edición conjunta de sus libros anteriores, inspiradora de tantos músicos—, su empresa Harry \& Co., su primer amor, su relación con August Schlegel en la Universidad de Bonn, su contacto con Goethe en Weimar, su asistencia a las clases de Hegel en Berlín, algunas "capitulaciones" (p. 313) —así llama Sacristán al seguramente forzado

\footnotetext{
${ }^{3}$ En Alemania, el contexto de esa conmemoración era bastante difícil porque aún se recordaba que las obras de Heine habían sido quemadas por los nazis en tanto que escritas por un judío, a pesar de la importancia que tuvieron para la forja ideológica de Alemania los poemas heineanos; mientras que, por otro lado, el marxismo más o menos dominante era tradicionalmente crítico del romanticismo, a pesar de ser Marx un romántico poeta político — dicho sea de paso, Marx nunca secundó las críticas puritanas hacia Heine que surgieron en los primeros círculos comunistas.

${ }^{4}$ En realidad sorprende que Sacristán seleccione en sus Obras de Heine, sólo narraciones, ensayos y memorias, es decir, el Heine prosista, aunque gran parte de su estudio lo dedique al análisis de su obra poética. Acaso sea bueno recordar que la recepción de Heine en torno a su centenario tuvo una particularidad interesante: mientras la Alemania capitalista se interesaba por su poesía, la Alemania comunista lo hacía por su prosa.
} 
bautismo de Heine para hacerse protestante, sintiendo "por ver primera el abismo de la mentira en su propia vida, la falla mortal del no coincidir ya consigo mismo" (p. 314) - y sus fallidos intentos de ser profesor, funcionario o periodista. En este recorrido, llama la atención el juicio negativo que le merece a Sacristán el Buch der Lieder heineano ${ }^{5}$ : "el fácil talento verbal, (...), la exacerbación de todas las exageraciones románticas, la incoherencia con cual, desde muy joven, escribe y publica Heine versos de acrítico romanticismo y contundentes parodias realistas de la falsedad romántica, todos esos elementos de falsedad enterraron muy pronto su poesía más famosa" (p. 317); “tono falso y vacio” (p. 318), “dejadez y facilonería" (ib.).

Heine iba camino de París al calor de su admirada revolución, la de 1831, que había derrocado al rey por derecho divino, Carlos X, y puesto en su lugar al rey por gracia de la burguesía, Luis Felipe de Orleans. Este compromiso con la revolución fue vivido y presentado por Heine como el triunfo de lo político sobre lo poético — triunfo que Sacristán aplaude- y el contexto en el que ven la luz Zur Geschichte der Religion und Philosophie in Deutchland [Sobre la historia de la religión y la filosofía en Alemania] y Die romantische Schule [La escuela romántica]. De hecho, en el prólogo de 1837 para su Buch der Lieder admite su cansancio y reconoce que la musa poética le ha abandonado. Pero las crónicas que Heine publica en el Allgemeine Zeitung (Ausburg) acaban molestando dado que, en su genérica defensa de la libertad, no solo muestra su odio a los nobles y al clero, sino también, y de forma más intensa aún, a burgueses, banqueros y empresarios; da, además, la bienvenida al emergente socialismo, a la vez que se presenta como moderado y monárquico. Finalmente la Gaceta General de Ausburgo lo despide, perdiendo Heine así también, es de suponer, la asignación que le pagaba la República francesa por tal cometido. Con todo, el año de su crack total fue 1848: a la nueva revolución —que, esta ya sí, le asusta — se suma su quiebra financiera, el conocimiento público de que recibía dinero de Francia y la explosión de su larvada enfermedad, que le obliga a vivir en cama hasta el final de sus días.

Heine cumple así el repetido ciclo de juventud revolucionaria y sensualista, y final conservador y neoconverso. Algo que siempre ha dado mucho que pensar

\footnotetext{
${ }^{5}$ Es probable que ese juicio negativo refleje el tono general de una Europa en plena Guerra Fría. A diez años del final de la Segunda Guerra Mundial, no era buen momento para lo romántico. Pero ya estaba gestándose la explosión juvenil del 68, todo un triunfo del romanticismo, movimiento al que, acaso inconscientemente, contribuiría la publicación de la Obras de Heine en España. Cf. p. 318, nota 13.
} 
y cuya elucidación es el leit-motiv del ensayo sacristaniano. A Sacristán le parece plausible la explicación psicologista del ciclo heineano: desde su encumbramiento en los años treinta a su decadencia y ruina finales ${ }^{6}$; pero cree que, en todo caso, esa "vileza egoísta" (p. 336) se puede retrotraer a su juventud. De modo que el derrumbe final, a su juicio, no se debió a causas externas sino a causas internas, casi constitutivas: "Lo que Heine consideró su doctrina en la época floreciente de su vida era un conjunto de inseguras vaguedades, hemiconceptos y gestos de idea, meros ecos de estados de ánimo. En eso no puede asentarse firmemente ninguna consciencia, por aguda que sea. El secreto de las desaventuras de la consciencia de Heine está precisamente ahí, (...). La enfermedad, la ruina y el miedo triunfaron porque no tenían enfrente más que una frágil choza ideológica en la que nunca habitó segura la consciencia de Heine" (p. 344). Las tres corrientes que confluyeron en Heine — romanticismo, hegelianismo, saint-simonismo- no dieron lugar a una síntesis arquitectónica. De ahí la derrota de la consciencia heineana.

Llegados a este punto, Sacristán asesta el golpe definitivo, que no va dirigido sólo a Heine sino a toda una corriente de compromiso político a medias: "Esa irrealidad, ese carácter ilusorio de la aspiración revolucionaria, que tiene en Heine un representante característico, va a ser desde entonces destino del intelectual inconformista que no llegue a insertarse orgánicamente en el tronco de los reales portadores de la historia moderna desde 1830 y 1848. Heine es también en esto uno de los grandes introductores del mundo contemporáneo, y [de] la ambigüedad provocada por la presencia simultánea en él de una aspiración revolucionaria y una incapacidad de asumir orgánicamente un lugar en la lucha de los pueblos" (p. 350). Me permito destacar la doble ocurrencia del término orgánico en esta cita porque apunta a la decisiva cuestión a la que se enfrentaba el propio Sacristán, con implicaciones en toda su generación: militar o no militar. Ha de recordarse su propia militancia clandestina en el PSUC y el PCE por esos años y las discrepancias posteriores que le llevaron, primero, a dejar sus cargos y, a finales de los años setenta, a abandonar la militancia. Esa lucha o alternativa entre la libertad subjetiva e idealista —irónica, utópica, ciclotímica, anarquizante, Berlín oeste, por

\footnotetext{
${ }^{6}$ Entre otras citas con las que Sacristán ilustra esta ruina intelectual se lee la siguiente, procedente de las Confesiones (1854): "Mientras tales doctrinas [el panteísmo y el ateísmo] fueron propiedad secreta de una aristocracia de iniciados (...) me conté yo también entre los imprudentes espritsforts. Pero cuando observé que la grosera plebe, el Juan Lanas, empezaba también a discutir esos temas (...) se me abrieron de repente los ojos, y mi ateísmo, gracias a Dios, se volatilizó en el acto (...); comprendí, en efecto, que el ateísmo ha concertado una alianza más o menos secreta con el más espantoso y nudo, con el más desnudo y común comunismo".
} 
poner un ejemplo_- por un lado, y la sujeción a la línea ideológica y estilística — realista, materialista, canónica, orgánica, en este caso Berlín este- marcada por el Partido, comunista se entiende, por otro, creo que es la que determina el duro juicio de Sacristán sobre Heine. En breve, parece objetarle que no se sumara a la naciente Liga de los Comunistas, con la que ya colaboraba su joven paisano $\mathrm{K}$. Marx, con quien ya tenía contactos personales y de colaboración periodística. Ahí hubiera encontrado el armazón ideológico que hubiera alimentado y fortalecido orgánicamente sus débiles raíces.

Cabe pensar, con todo, que quizá unos años más tarde el juicio de Sacristán, alimentado en este caso por sus decepciones políticas, hubiera sido distinto. Sacristán busca coherencia en Heine con una medida de coherencia más estrecha que la puesta en práctica por el poeta, poeta al fin; pero Heine era coherente en su ductilidad, sólo que su medida era más amplia; ahora bien, siempre tuvo claros sus límites: nunca materialismo a secas, nunca Locke (ni La Mettrie, ni d'Holbach), nunca industrialismo, de Voltaire sólo un poco, por un lado, y, por otro, nunca jesuitismo, nunca catolicismo político, nunca religión racional, nunca muerto medievalismo. Creo por tanto que el enfoque hermenéutico sacristaniano no ha aguantado bien el paso del tiempo. Lo romántico, en cualquier caso, recuperó su espacio y Heine, todo un clásico, se benefició de ello. También sus contradicciones han encontrado juicios más benévolos. De hecho, Jesús Munárriz, en el breve prólogo a su antología de cincuenta poemas que titula Radikal [Hiperión, 2008], escribe: "En la amplia obra poética de Heinrich Heine conviven diversas líneas y temáticas, que se entremezclan y enriquecen mutuamente, (...), en Alemania, cuento de invierno conviven en magnífico equilibrio todas las facetas del poeta Heine" (pp. 9, 10). Respecto al exceso de biografismo, José Luis Reina Palazón escribe, en la presentación de su edición bilingüe de El libro de las canciones [Linteo, 2009] que ha sido un "malentendido" (p. 13) que ha impedido ver lo más importante: la ironía, las citas, las imitaciones, el gusto por la variación y la agudeza; así se descubre "la gran distancia entre el mundo de la poesía y el real" (p. 15).

Si retrocedemos ahora ciento treinta años, nos situamos finalmente en el momento en que Heine da a conocer su interpretación de la literatura, la religión y la filosofía escritas en alemán, gran parte de la cual él conoce de primera mano. Son textos por tanto de disputa, si no de combate, y de construcción de la autoconsciencia francesa y alemana. Desde Francia, su ala conservadora - Madame de Stäel y Victor Cousin_ - habían dado a conocer la parte de la cultura alemana que les servía como modelo que oponer al auge de las corrientes culturales bajo el Imperio napoleónico. Heine ridiculiza tales intentos y se propone, en tanto 
que poeta alemán y mejor conocedor de la filosofía alemana, como intérprete alternativo. En "La escuela romántica" — “alemana" habría que añadir para evitar confusiones ${ }^{7}$ - presenta la moda que habían promocionado los hermanos Schlegel, August y Friedrich, a la que perteneció el mismo Heine ${ }^{8}$. El texto tiene por tanto mucho de autobiografía encubierta, cargada de ironía y distancia puesto que en el momento en que escribe Heine ya se considera el enterrador de esa secta. Es en ese sentido en el que el poeta renano se presenta como antirromántico, como harto del romanticismo de los Schlegel, el que, como reacción contra la ilustración y el neoclasicismo, defendió el culto a la Edad Media, al cristianismo como triunfo del espíritu sobre la carne, al ciclo de los Nibelungos ${ }^{9}$ y del Libro de los Héroes, a la caballería andante, a los Parsifal, Lohengrin, Tristán e Isolda, al simbolismo, a la arquitectura gótica y a lo popular. Esta escuela encarnó el patriotismo pangermano contrario a Napoleón y al cosmopolitismo y fue la que tradujo al alemán las obras de Shakespeare, Calderón y el Don Quijote — con prólogo de Heine, fechado en París en 1837- para contrarrestar las traducciones de Homero y de los clásicos latinos, que apoyaban el paganismo. Gran parte de sus integrantes, además, se hicieron católicos. Tras lo cual se produjo una reacción liberal-protestante contra lo que se consideraba el triunfo del jesuitismo. Y ahí estaba el joven Heine, junto a otros jóvenes, dando lugar a la Joven Alemania.

En el ensayo heineano es memorable la recreación de la lucha entre las dos Alemanias en las figuras de Johan H. Voss y de Fritz Stolberg — pp. 188-193-. Gran atención merece el longevo y multifacético Goethe, adorado por unos —a los que Heine considera papanatas - y denigrado por otros — porque los tenía, aunque ahora cueste creerlo-, pero al que Heine nunca pierde el respeto; así, confiesa: "quiero, empero, decir una vez más en descargo mío que nunca ataqué en Goethe al poeta, sino sólo al hombre. Nunca he denigrado sus obras. Nunca he podido ver en ellas defectos, como esos críticos que con pulidísimos anteojos reproducen las manchas de la luna" (p. 203). Con todo, considera que la poética y la vida de Goethe tienen un efecto paralizante en la juventud; muy otro es el caso de Schiller, en quien destaca su aspecto revolucionario y de defensor de la libertad y el progreso. El grueso del texto heineano lo ocupa, sin embargo, el pormenorizado repaso a las obras de los fundadores, los hermanos Schlegel —iqué derroche de

\footnotetext{
${ }^{7}$ Recuérdese que cuando Heine da a conocer su ensayo ya han muerto William Blake, Percy B. Shelley, John Keats y Walter Scott.

${ }^{8}$ Al fino olfato de Heine, no obstante, se le escapó Hölderlin, el gran ausente.

${ }^{9}$ cf. pp. 258-9, donde Heine alaba sobremanera este poema.
} 
ironía en estos retratos, casi caricaturas!_- Tieck — quien tradujo nuevamente El Ingenioso Hidalgo Don Quijote de la Mancha_, Novalis —-memorables son las páginas 247-250, donde Heine recrea cómo llegó a sus manos un ejemplar del Heinrich von Ofterdingen novaliano_- Hoffmann —incluido aunque no tuvo relación directa con los Schlegel_- Brentano —recogió y editó canciones populares alemanas de las que Heine escribe: "es imposible elogiar ese libro todo lo que se merece" (p. 254)—, Arnim - hay delectación en la detallada presentación heineana de los temas de un poeta que "no era un poeta de la vida, sino de la muerte" (p. 260)_- Werner, Fouqué, y Uhland —estos últimos los más representados, los más influyentes, los más populares_- Mención especial merece el capítulo III del libro tercero, donde Heine presenta a los poetas de la "Joven Alemania", cuyo precursor fue Jean Paul Friedrich Richter, los recentísimos Heinrich Laube y Karl Gutzkow; de ellos escribe que: "no quieren hacer tampoco ninguna diferencia entre vivir y escribir, ni separar jamás la política de la ciencia, el arte y la religión, y son al mismo tiempo artistas, tribunos y apóstoles" (p. 270).

Heine se incluiría en este último grupo, que se presenta como superador del movimiento romántico. De ahí la ambigüedad de título de antirromántico aplicado a Heine. A él ya no le dicen nada los temas típicos de esa corriente, en ese sentido la considera agotada, por lo que hay buscar caminos nuevos. Toda moda ha de dejar paso, de grado o por la fuerza, a la siguiente; de lo contrario, el ya entonces poderoso mercado editorial se quedaba sin mercancías nuevas que llevar a las ferias y ofrecer a sus clientes. Pero las sendas que abrió y recorrió Heine fueron también románticas, y en grado sumo. Queda así aclarada, espero, la paradoja planteada por el profesor Garrido. Con todo, lo paradójico es que el gusto cultivado por los hermanos Schlegel nunca desapareció, a pesar de lo que podía esperar Heine: pueden considerarse sus herederas las óperas de Wagner, que están vivas hasta el día de hoy; secuelas pueden considerarse también El señor de los anillos, los superhéroes, Harry Potter, Juego de tronos, Érase una vez y hasta la tribu urbana de los góticos. Heine se asocia sin embargo a la corriente romántica más extendida, caracterizada por su ductilidad. En ese sentido, romántico es el Rin renano, el Moncayo, R. Schuman, el magnífico relato darwiniano Diario del viaje de un naturalista alrededor del mundo (1836), todo Nietzsche, las vanguardias históricas, el 68, el arte contemporáneo, todo un subgénero novelístico, la posmodernidad, gran parte del cine hollywoodiense, Madonna, el culto al yo exacerbado y a la provocación en internet, y la adolescencia persistente. En definitiva, romántico es todo lo que toca el romántico. 
"La escuela romántica" presenta, además de las reseñadas, otras trazas llamativas. En sus retratos y caricaturas, Heine hace gala no sólo de su conocimiento de la cultura clásica, helenística sobre todo, sino también de la importancia que le concede a la religión, católica y protestante, y a la filosofía, sobre todo a Schelling. Allí donde una obra o un autor le entusiasman, enseguida Heine eleva el tono y plantea las grandes cuestiones religiosas y filosóficas. Es este un rasgo idiosincrático. Tales intereses y tales lecturas, le permitieron escribir "Sobre la historia de la religión y la filosofía en Alemania". En este ensayo Heine comienza hablando de Lutero y de la importancia de su traducción de la Biblia para el alemán, y, en general, el protestantismo para la filosofía alemana. Los grandes nombres de Leibniz, aunque no escribió en alemán, Wolff, Kant, Fichte, Schelling y Hegel pasan por estas páginas, de intención divulgativa pero repletas de iluminaciones, que resultaron seminales para las historias de la filosofía académicas posteriores. Heine asocia el protestantismo a la libertad y el catolicismo a la reacción; defiende el carácter constitutivamente filosófico de la lengua alemana; critica el deísmo ilustrado y defiende el panteísmo de Spinoza, encumbra a Kant como el padre de la filosofía alemana y muestra sus simpatías por la filosofía de la naturaleza schelliniana, etc. — sin olvidar las terribles páginas finales 157-160, casi proféticas, si es que hay profetas-. Es un texto, en definitiva, que merece un estudio en profundidad.

Daniel Moreno Moreno IES Miguel Servet, Zaragoza dmoreno@unizar.es 